\title{
El contenido de arcilla del suelo influye en el rendimiento de un cultivo de tomate (Solanum lycopersicum L.)
}

\section{Clay content of soil influence on yield of tomato (Solanum lycopersicum L.) crop}

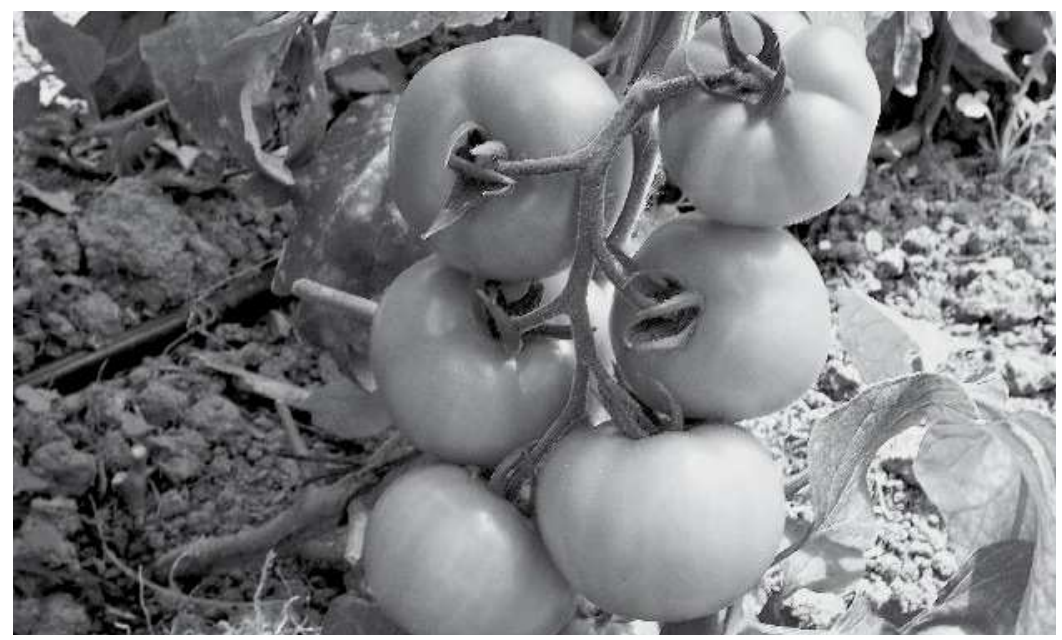

HELBER ENRIOUE BALAGUERA-LÓPEZ1,2

JAVIER GIOVANNI ÁLVAREZ-HERRERA ${ }^{1}$

GLORIA ESPERANZA MARTÍNEZ-ARÉVALO'

WILLIAM ALBERTO BALAGUERA ${ }^{1}$

Racimo de tomate híbrido Granitio completamente formado.

Foto: R. Quintana

\section{RESUMEN}

El tomate es la hortaliza de mayor importancia a nivel mundial por su alto consumo y área cultivada. Aunque esta planta se adapta a un amplio rango de suelos no se ha determinado el contenido de arcilla en el que se obtiene mayor producción de fruto y de mayor calidad comercial. Por tanto, el objetivo de este trabajo fue evaluar seis contenidos de arcilla en un Typic Haplustalf (1\%, 10\%, 20\%, 30\%, 40\% y 50\%) sobre el rendimiento y algunas variables fisiológicas de plantas de tomate larga vida híbrido Granitio cultivadas en invernadero plástico. Se cosecharon los frutos durante 60 días y se clasificaron por calidades comerciales. Al final de la cosecha se midió altura, área foliar, fitomasa fresca y seca de raíz, tallo y hojas. Únicamente se presentaron diferencias estadísticas en la calidad segunda, mas no en el rendimiento total ni en las demás calidades. No obstante, el $10 \%$ de arcilla favoreció el mayor rendimiento y calidad extra. La altura de plantas, área foliar, fitomasa fresca y seca de hojas presentaron diferencias estadísticas, siendo la mayor respuesta con 30\% de arcilla. Es recomendable sembrar plantas de tomate en suelos con contenidos de arcilla hasta el $50 \%$, con rendimientos similares, aunque con $10 \%$ hay mayor respuesta en producción.

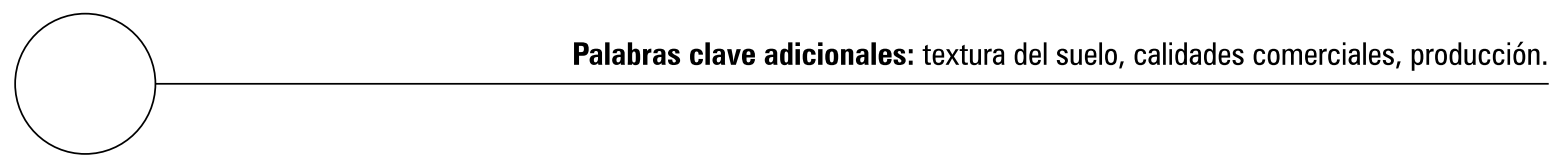

1 Grupo de Investigaciones Agrícolas, Facultad de Ciencias Agropecuarias, Universidad Pedagógica y Tecnológica de Colombia, Tunja (Colombia).

2 Autor para correspondencia. enrique_balaguera@yahoo.com 


\section{ABSTRACT}

Tomato is the most important vegetable crop globally for its high consumption and cultivated area. Although this plant is adapted to a wide range of soils, the contents of clay in which it gets increased fruit production and the highest commercial quality have not been determined. Therefore, the objective of this study was to evaluate the effect of six clay contents in a Typic Haplustalf soil $(1 \%, 10 \%, 20 \%, 30 \%, 40 \%$, and $50 \%$ ) on yield and some physiological variables of tomato plants long life Granitio hybrid growing in plastic greenhouse. The fruits were harvested during 60 days and classified according to commercial qualities. At the final harvest the height, leaf area, fresh and dry mass of root, stem and leaves were measured. There were statistical differences observed only in second quality, but neither over total performance and other qualities. However, $10 \%$ clay favored the highest yield and extra quality. The plant height, leaf area, fresh and dry weight of leaves presented statistical differences with the greatest response of $30 \%$ clay. It is advisable to plant tomato crops in soils with clay contents up to $50 \%$, with similar yields, although with $10 \%$ clay there was the highest production.

Additional key words: soil texture, comercial qualities, production.

El tomate (Solanum lycopersicum L.) es la hortaliza más importante a nivel mundial ya que presenta la mayor área cultivada, mayor consumo y el valor de esta hortaliza es superior al de cualquier otra (Escobar y Lee, 2001; Peralta y Spooner, 2007). En el año 2008, a nivel mundial se tenían sembradas 5.227 .883 ha con una producción de 129.649.883 t (FAO, 2009), mientras que en nuestro país, para ese mismo año, el área cultivada fue de 14.855 ha con una producción de 455.693 t. En el departamento de Boyacá se sembró el $12,99 \%$ del área total cultivada en tomate a nivel nacional, con un rendimiento de 65,6 t ha-1 , y lo convierte en el departamento con mayor productividad del país (Agronet, 2009). Debido a la importancia económica, el tomate es una de las especies más cultivadas bajo superficies cubiertas por plástico, y es un sistema de gran relevancia social ya que se lleva a cabo generalmente con mano de obra familiar en pequeñas áreas (Radin et al., 2003).
Gran parte de las investigaciones están encaminadas a que el cultivo de tomate se realice bajo condiciones de hidroponía (Logendra y Janes, 1999; Fernandes, 2000; Okano et al., 2000; Sakamoto et al., 2000; Fernandes et al., 2002), sin embargo, el cultivo en suelo es el más difundido por ser un sistema mas práctico, económico y por estar al alcance de todos los productores.

La disminución en la productividad de los cultivos ha sido atribuida generalmente al deterioro de la fertilidad química del suelo (Ali, 1998), dejando relegada la importancia de la fertilidad física (Acharya et al., 1998) debido a que el concepto de fertilidad viene siendo enfocado más a la presencia de nutrientes en el suelo, aunque su disponibilidad es una función del ambiente físico del mismo. Este ambiente influye en la naturaleza de las reacciones químicas y biológicas necesarias para el óptimo desarrollo de las plantas (Sharma et al., 2003). Por su parte, Mejía (1975) 
afirma que directa o indirectamente, la proporción, composición y estructura de las diferentes fracciones como arena, limo y arcilla que integran el suelo, determinan gran parte de sus características químicas y físicas, y por tanto, su fertilidad.

De las partículas minerales del suelo, la fracción arcilla se caracteriza por su alta actividad química, principalmente porque es de naturaleza anfótera, que le confiere capacidad para atraer tanto cationes como aniones, aunque es más importante por su alta capacidad de retener e intercambiar cationes (Mejía, 1975). Además es un agente cementante que favorece la formación de agregados en el suelo (Bullinger-Weber et al., 2006). Brown (1977) afirma que la influencia que ejercen las arcillas dependiendo de su composición mineralógica y la proporción en que ella interviene en los suelos incluye la velocidad de infiltración, el drenaje interno y la capacidad de retención de humedad, siendo más difícil el movimiento del agua y el aire en el suelo cuando el contenido de arcilla es mayor, pero la retención de agua y nutrientes es mayor (Brady, 2004).

El tomate puede ser cultivado en un amplio rango de tipos de suelos (Kinet y Peet, 1997). Sin embargo, el suelo ideal para este cultivo debe ser bien drenado pero a la vez capaz de retener humedad. La aireación es uno de los factores físicos más importantes que puede limitar el desarrollo de los sistemas radiculares de las plantas, el crecimiento y la producción de los cultivos (Czyz y Tomaszewska, 1994). Según Escobary Lee (2001), el tomate necesita de suelos bien aireados, con alta capacidad de almacenamiento de agua útil $y$ con un buen nivel de fertilidad. Aunque bajo condiciones de invernadero se puede cultivar en una gran variedad de suelos, se prefiere aquellos de texturas francas con altos contenidos de materia orgánica. Adicionalmente, es necesario que se tenga buen drenaje, debido a que las raíces de las plantas de tomate no toleran excesos de agua.

El tomate es una de las plantas más sensibles al exceso de humedad y pobre suministro de oxíge- no en el suelo (Bradford y Yang, 1981). Periodos de excesivo contenido de agua en el suelo tienden a generar plantas con follajes más reducidos y con producciones mínimas. Las plantas requieren un adecuado contenido de oxígeno para la respiración de las raíces y cumplir con sus funciones metabólicas (Barrett-Lennard, 2003).

No obstante, no se sabe con certeza cuál es el contenido de arcilla que mejor favorece la producción de frutos en las plantas de tomate. Por tanto, el objetivo de este trabajo fue evaluar diferentes porcentajes de arcilla del suelo para determinar su efecto en el rendimiento de un cultivo de tomate larga vida bajo condiciones de cubierta plástica.

\section{MATERIALES Y METODOS}

El estudio se realizó en el municipio de Sutamarchan (Boyacá) ubicado a 15³7'42,7" N y $73^{\circ} 38^{\prime} 12,8^{\prime \prime}$ W. En un lote bajo cubierta plástica de $1.100 \mathrm{~m}^{2}$ se sembró un cultivo comercial de tomate larga vida híbrido Granitio, ubicado a $2.315 \mathrm{msnm}$. Se presentó una temperatura interior promedio de $22^{\circ} \mathrm{C}$ y una humedad relativa del $80 \%$. Los análisis de suelos se llevaron a cabo en el laboratorio de suelos, mientras que las pruebas fisiológicas se hicieron en el laboratorio de Fisiología Vegetal de la Facultad de Ciencias Agropecuarias de la Universidad Pedagógica y Tecnológica de Colombia, Tunja.

Se realizó la descripción del perfil del suelo encontrado en el sitio de trabajo, basados en los resultados físicos y químicos del suelo que se expresaron en las tablas 1 y 2 y según las claves taxonómicas determinadas por USDA (2006), el suelo correspondió a un Typic Haplustalf, el cual se caracteriza por tener suelos minerales con horizontes de iluviación de arcillas y saturación relativamente alta en profundidad, con humedad suficiente para el desarrollo de los cultivos. El clima de la zona es frío seco, tiene un régimen de temperatura isofrígido y un régimen de humedad ústico, con un epipedón ócrico y un endopedón argílico. 


\begin{tabular}{|c|c|}
\hline Propiedad & Descripción \\
\hline $\begin{array}{l}\text { Color } \\
\text { En húmedo } \\
\text { En seco }\end{array}$ & $\begin{array}{l}\text { Dark greyish brown }(2.5 \text { y } 4 / 2) \\
\text { Light olive brown }(2.5 \text { y } 5 / 3)\end{array}$ \\
\hline Textura & Franco arcillosa (FAr) \\
\hline $\begin{array}{l}\text { Estructura } \\
\text { Clase } \\
\text { Grado }\end{array}$ & $\begin{array}{l}\text { Tipo bloques angulares y subangulares } \\
\text { Mediana a gruesa } \\
\text { Fuerte }\end{array}$ \\
\hline $\begin{array}{l}\text { Consistencia } \\
\text { En seco } \\
\text { En húmedo } \\
\text { En mojado }\end{array}$ & $\begin{array}{l}\text { Dura } \\
\text { Friable } \\
\text { Pegajosa }\end{array}$ \\
\hline Porosidad & Moderada \\
\hline $\begin{array}{l}\text { Macroorganismos } \\
\text { y raíces }\end{array}$ & Pocos \\
\hline $\mathrm{pH}$ & 6,8 \\
\hline $\begin{array}{l}\text { Reacción } \\
\mathrm{NaF} \\
\mathrm{HCl} \\
\mathrm{H}_{2} \mathrm{O}_{2}\end{array}$ & $\begin{array}{l}\text { Media } \\
\text { No reactivo } \\
\text { Baja }\end{array}$ \\
\hline
\end{tabular}

Dentro del invernadero se tomaron 66 puntos en una red rectangular de $4 \times 4 \mathrm{~m}$, con el fin de realizar el muestreo de suelos. En cada punto se tomaron cinco submuestras una central y cuatro separadas a $30 \mathrm{~cm}$ en cada eje cardinal, siguiendo la metodología propuesta por Martínez y Zinck (1994), a una profundidad de entre 0 y $20 \mathrm{~cm}$; luego se mezclaron y se obtuvo una muestra compuesta de $100 \mathrm{~g}$ con la cual se determinó la textura por el método del hidrómetro de Bouyoucus (IGAC, 2006). Con los resultados obtenidos del contenido de arcilla se plantearon seis tratamientos que fueron $1 \%, 10 \%, 20 \%, 30 \%$,
$40 \%$ y $50 \%$. Se utilizó un diseño completamente el azar con ocho repeticiones.

A los 30 d después del muestreo de suelos se sembraron las plantas de tomate, se llevaron a cabo todas las labores culturales como manejo de plagas, podas, suministro de riego y fertilización mediante fertirrigación con sistema de riego por goteo. El plan de fertilización se hizo a diario con base en el análisis de suelos, aplicando dos y tres riegos diarios dependiendo de la etapa fenológica y las condiciones climáticas. A los $60 \mathrm{~d}$ después del trasplante se inició la cosecha y se recolectaron los frutos dos veces por semana durante dos meses. Se podó el meristemo apical de las plantas cuando formaron el séptimo racimo.

Se calculó el rendimiento del cultivo $\left(\mathrm{kg} \mathrm{ha}^{-1}\right)$, se determinó la producción por planta, y se extrapoló a hectárea, teniendo en cuenta que la densidad de plantación fue de 41.666 plantas/ha; rendimiento por calidades comerciales $\left(\mathrm{kg} \mathrm{ha}^{-1}\right)$, de acuerdo al diámetro del fruto (tabla 3), medidas con una plantilla de calibres para tomate, para esto se midieron y pesaron los frutos recolectados; área foliar, mediante el medidor portátil de área foliar CI-202 Seedmech; masa fresca de raíz, tallo y hojas; masa seca de raíz, tallo y hojas después de someter las plantas a $70^{\circ} \mathrm{C}$ durante $48 \mathrm{~h}$ en estufa de secado.

Se realizó la prueba de normalidad con el Test de bondad de Kolmogorov-Smirnov y la prueba de homogeneidad de varianzas con el test de Le-

Tabla 2. Resultados del análisis fisicoquímico del suelo.

\begin{tabular}{|c|c|c|c|c|c|c|c|c|c|c|}
\hline \multicolumn{11}{|c|}{ Resultados de propiedades físicas del suelo } \\
\hline \multicolumn{2}{|c|}{ Horizonte } & \multicolumn{3}{|c|}{ Granulometría } & \multirow{2}{*}{\multicolumn{2}{|c|}{$\begin{array}{c}\text { Clase } \\
\text { textural }\end{array}$}} & \multirow{2}{*}{$\mathrm{pH}$} & \multicolumn{2}{|c|}{ Densidad $\left(\mathrm{g} \mathrm{cm}^{-3}\right)$} & \multirow{2}{*}{ Porosidad (\%) } \\
\hline Pprof $(\mathrm{cm})$ & Nomenclatura & $A$ & $L$ & $A$ & & & & Aparente & Real & \\
\hline $0-80$ & Bt1 & 26 & 51 & 23 & & $\operatorname{Ar}$ & 6,8 & 1,09 & 2,48 & 55,65 \\
\hline \multicolumn{11}{|c|}{ Resultados de propiedades químicas del suelo } \\
\hline \multicolumn{2}{|c|}{ Horizonte } & \multicolumn{5}{|c|}{ Complejo de cambio $\left(\mathrm{Cmol}^{+}\right)$} & \multirow{2}{*}{$\mathrm{Al}^{3+}$} & \multirow{2}{*}{$\% S A L$} & \multirow{2}{*}{$\mathrm{P}_{2} \mathrm{O}_{5}$} & \multirow{2}{*}{$\mathrm{CO}(\%)$} \\
\hline Pprof $(\mathrm{cm})$ & Nomenclatura & $\mathrm{ClC}$ & $\mathrm{Ca}$ & $\mathrm{Mg}$ & K & $\mathrm{Na}$ & & & & \\
\hline $0-80$ & Bt1 & 23,98 & 15,91 & 6,53 & 0,16 & 0,147 & 0 & 0 & 23 & 1,1 \\
\hline
\end{tabular}


Tabla 3. Clasificación de los frutos de tomate de acuerdo al calibre.

\begin{tabular}{|l|c|}
\hline \multicolumn{1}{|c|}{ Clasificación } & Diámetro del fruto $(\mathrm{mm})$ \\
\hline Calibre 1 (extra) & $>82$ \\
\hline Calibre 2 (primera) & 67 a 82 \\
\hline Calibre 3 (segunda) & 57 a 67 \\
\hline Calibre 4 (tercera & 47 a 57 \\
\hline Calibre 5 (cuarta) & 40 a 47 \\
\hline
\end{tabular}

Fuente: Adaptado de Escobar y Lee, 2001.

vene, con el fin de utilizar estadística paramétrica. Se hizo un análisis de varianza (Anova), se utilizó la prueba de comparación múltiple de Tukey con una confiabilidad de $95 \%$, y se determinó la prueba de componentes principales para establecer las variables más importantes y la correlación entre ellas, utilizando el software SAS v.8e (Cary, NC).

\section{RESULTADOS Y DISCUSIÓN}

Todas las variables superaron el supuesto de normalidad y homogeneidad de varianzas, por tanto, fue posible la utilización de estadística paramétrica. Se encontró que el contenido de arcilla no tiene un efecto representativo en la producción del tomate, ya que se determinó hasta el quinto eje de los componentes principales y solo explicó $78,99 \%$ de la varianza. Del mismo modo, la correlación con las calidades comerciales y el rendimiento del cultivo no fue representativa, teniendo para el total de la producción 0,82, calidad primera 0,18 , segunda 0,22 , tercera 0,16 y para la calidad extra la relación fue inversa con 0,37 .

Asimismo, solo se presentaron diferencias estadísticas $(P \leq 0,01)$ para la calidad segunda, favorecida por un $20 \%$ de arcilla en el suelo, mientras que la calidad tercera se vio beneficiada por $30 \%$ de arcilla. El contenido de arcilla en un $10 \%$ es el más adecuado para el rendimiento total pero sin diferencias estadísticas (figura 1). No obstante, en la producción de tomate el objetivo es obtener frutos del más alto calibre de calidad extra y primera, debido a que tienen mayor precio en el mercado, y el peso del fruto es mucho mayor que las demás categorías, este contenido de arcilla fa-

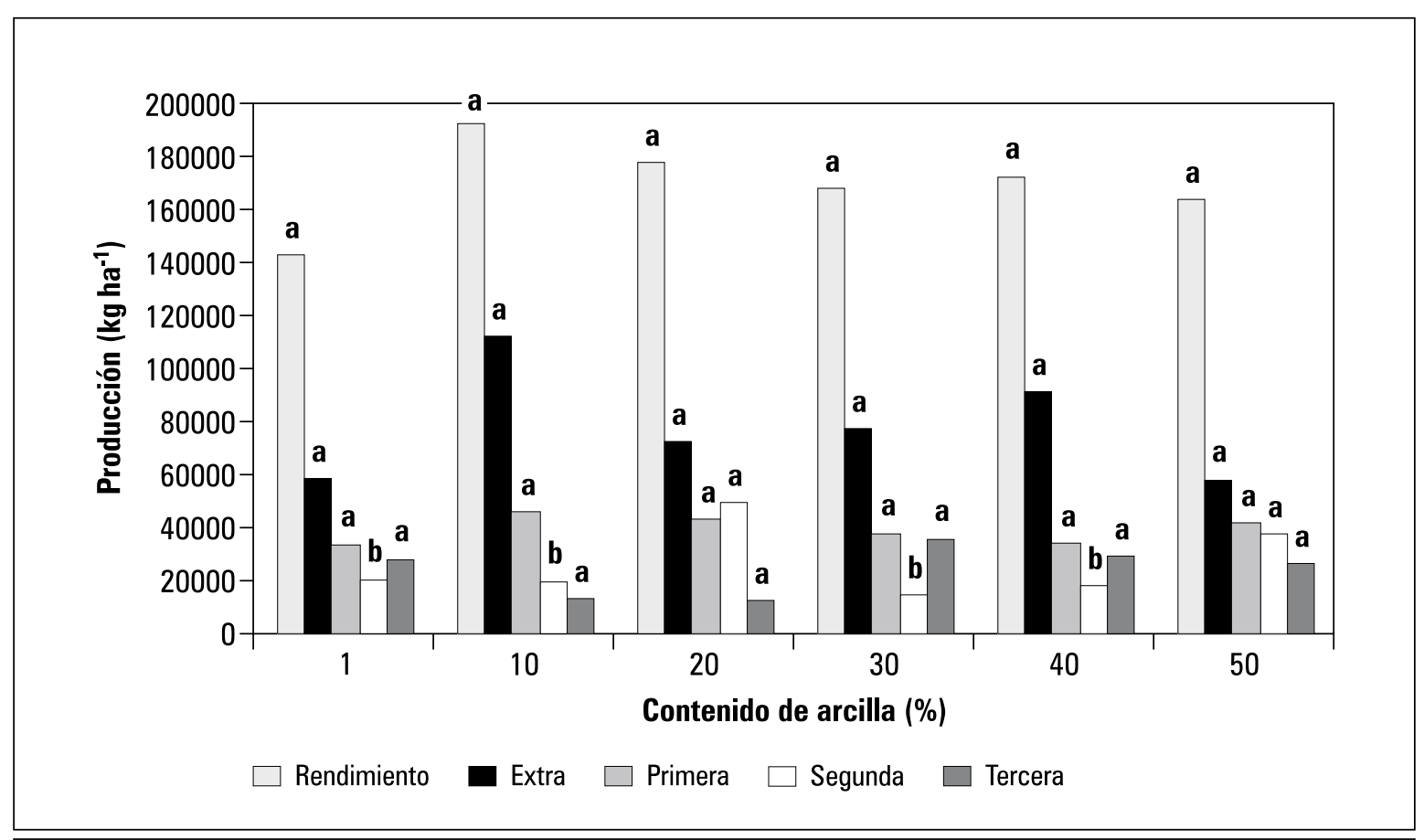

Figura 1. Rendimiento de plantas de tomate larga vida híbrido Granitio bajo el efecto del contenido de arcilla del suelo. Promedios con letras distintas en la misma serie indican diferencia significativa según la prueba de Tukey $(P \leq 0,05)$. 
vorece el rendimiento total con una correlación directa de 0,90 y 0,06 respectivamente.

El tomate tiene altos requerimientos de agua tanto en cantidad como en la frecuencia de suministro y van desde $0,6 \mathrm{~L} \mathrm{~m}^{-2} \mathrm{~d}^{-1}$ en estado de plántula a $6,5 \mathrm{~L} \mathrm{~m}^{-2} \mathrm{~d}^{-1}$ posterior al inicio de la cosecha. Además no se debe dejar que el suelo se seque demasiado y luego aplicar grandes cantidades de agua, pues esto ocasiona daños en las plantas y agrietamiento en los frutos (Medina et al., 2001). Lo anterior depende del sistema de riego y de la textura del suelo. Aunque con el sistema de riego por goteo se suministra el agua con alta eficiencia, el contenido de arcilla ayuda a mantener la humedad en el suelo para luego hacerla disponible gradualmente, evitando así los cambios bruscos de humedad.

Según Bresler (1977) mantener el potencial hídrico del suelo elevado se refleja en una mayor producción. Por otro lado, la aireación de un suelo depende de la distribución y tamaño de los poros (Richards, 1983) y es un factor crítico para el desarrollo de las raíces. En la mayoría de las especies, el espacio poroso ocupado por el aire no debe ser inferior a 10\% (Richards, 1983), por lo cual, el riego diario en suelos de textura fina, con problemas de compactación, puede provocar problemas en el desarrollo de raíces (INIA-ODEPA, 2000). Por lo que el efecto del riego por goteo es más favorable en suelos con bajo contenido de arcilla, con predominio de texturas gruesas, de baja capacidad de retención de humedad y buenas condiciones de aireación (Bresler, 1977), razón por la cual, el suelo con $10 \%$ de arcilla mostró mayores rendimientos en el cultivo de tomate.

Respecto al almacenamiento de nutrientes, el contenido de arcilla favorece la retención de los nutrientes (Mejía, 1975), sin embargo, mediante fertirrigación son suministrados los nutrientes necesarios para el cultivo, no obstante, con bajos contenidos de arcilla los nutrientes son fácilmente lixiviados y poco aprovechados por la planta, lo que redunda en pérdidas económi- cas (Escobar y Lee, 2001) y de producción, y es por eso que el rendimiento más bajo se debió al suelo con $1 \%$ de contenido de arcilla. Además, la fracción arcilla permite mayor disponibilidad de $\mathrm{K}$, el cual es considerado el elemento dominante en la producción de tomate (Huett y Dettmann, 1988) ya que actúa como activador enzimático, en la fotosíntesis, osmoregulación y transporte floemático (Marschner, 2002), lo que determina la producción final.

Cuando en el suelo domina la fracción arcilla, en la porosidad total del suelo hay más cantidad de microporos que cuando domina la fracción arena. En este caso existe una gran cantidad de macroporos en el espacio poroso. Lo anterior se comprende claramente, si se piensa que entre las microscópicas partículas de arcilla los espacios son pequeños. En cuanto a la magnitud de la porosidad total, es mayor cuando en la textura dominan las fracciones finas que cuando dominan las gruesas, por lo que los suelos arcillosos poseen más porosidad total que los arenosos (Brady, 2004).

Sin embargo, los microporos son los encargados de almacenar agua mientras que los macroporos están más implicados con el crecimiento radicular, por lo cual, suelos con predominio de arcilla afectan de forma negativa el sistema radicular (Brady, 2004) y por ende la producción (Czyz y Tomaszewska, 1994), pues las raíces de muchos cultivos necesitan un buen suministro de oxígeno para satisfacer los requerimientos de agua y nutrientes que necesita el resto de la planta (Meek et al., 1983). A pesar de esto, con contenidos de arcilla de 1 a $50 \%$ no se ve afectado negativamente el rendimiento del cultivo de tomate.

Por otro lado, las producciones obtenidas estuvieron cercanas a las $200 \mathrm{t} \mathrm{ha}^{-1}$, rendimientos considerables teniendo en cuenta que el rango para tomate larga vida está entre 110 y 388 t ha-1 por ciclo y la producción anual sobrepasa las 500 t ha ${ }^{-1}$, de tal manera que una planta tiene la capacidad de producir $24 \mathrm{~kg}$ (Ho, 1984). Además, 
el tamaño del fruto de tomate está determinado por el número de células (Bohner y Bangerth, 1988; Ho, 1992), es así, que el contenido de $10 \%$ de arcilla posiblemente garantizó una disponibilidad adecuada de agua, oxígeno y nutrientes, que a la vez favorecieron una mayor división celular, por lo cual, la cantidad de tomate calidad extra fue considerablemente mayor.

En uva se encontró que el tamaño de las bayas es muy sensible a los cambios de humedad del suelo, generados por la inadecuada retención de humedad en suelos con predominio de arenas y por el humedecimiento parcial del sistema radicular (Selles et al., 2003). Glenn (2000) y Dry et al. (2001) postularon que un mejoramiento limitado del sistema radicular de las plantas podría afectar la respuesta fisiológica del cultivo por mecanismos que no son solamente de carácter hídrico, por ejemplo, la falta de oxígeno en las raíces reduciría la capacidad de producir y transportar citoquininas, otras hormonas, y nutrientes a la parte aérea de la planta, lo que afecta el tamaño del cultivo y su producción (Davies y Zhang, 1991).
En cuanto a las variables fisiológicas, se presentaron diferencias significativas en la altura de plantas de tomate, con el 30\% de arcilla se obtuvo mayor altura, pero la baja correlación ( $r=-$ 0,0725 ) hace pensar que el contenido de arcilla no tiene un efecto directo en la longitud del tallo de estas plantas. Caso similar se evidenció en el área foliar, pues tuvo baja correlación $(r=0,008)$ y también fue el contenido de arcilla del 30\% el encargado de expresar una mayor área foliar (figura 2). Este contenido de arcilla asegura una adecuada disponibilidad de agua y nutrientes que son tomados por la raíz y transportados a la parte aérea para que se lleve a cabo la fotosíntesis, aunque parece ser que en este tratamiento los fotoasimilados producidos en las hojas son transportados y acumulados en el tallo para inducir mayor altura, no siendo así para el rendimiento.

Las plantas de tomate larga vida de híbridos indeterminados pueden crecer hasta $9 \mathrm{~m}$ de altura y producir hasta 35 racimos en un periodo de 10 a 11 meses en condiciones de invernadero (Ho, 1984), sin embargo, en este estudio las plantas no
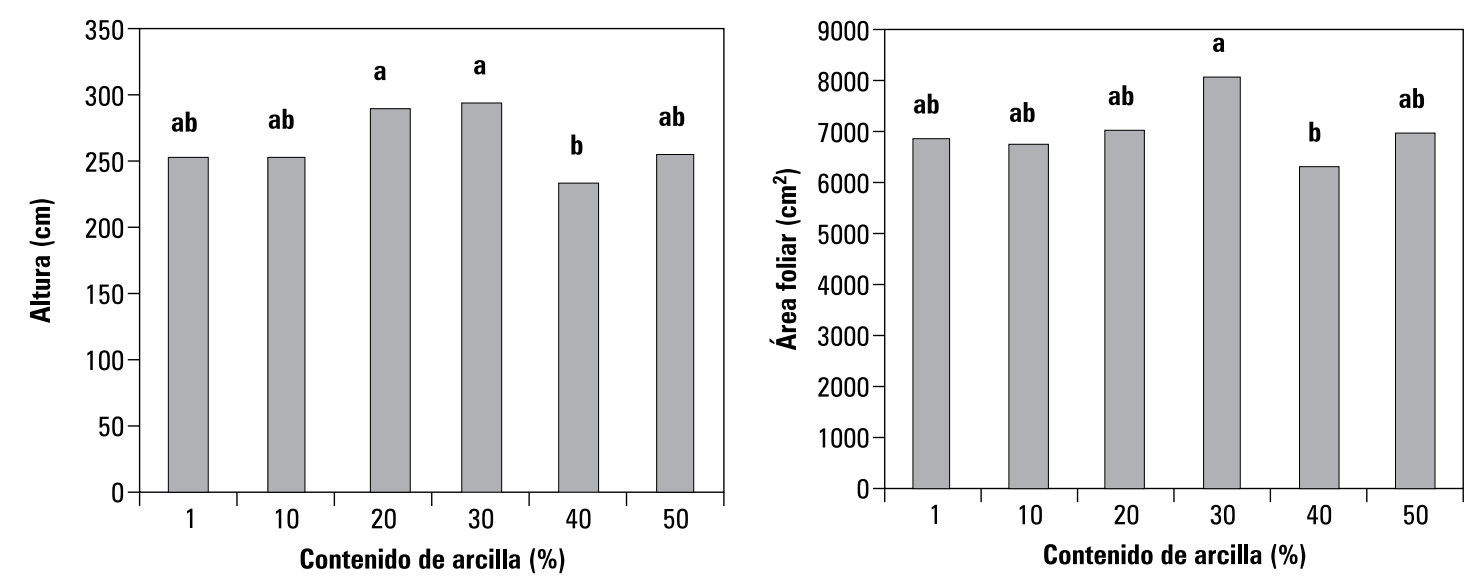

Figura 2. Altura y área foliar de plantas de tomate larga vida híbrido Granitio bajo el efecto del contenido de arcilla en el suelo. Promedios con letras distintas en la misma serie indican diferencia significativa según la prueba de Tukey $(P \leq 0,05)$. 
superaron los $3 \mathrm{~m}$ de altura, porque se hizo una poda en el meristemo apical cuando alcanzaron el séptimo racimo, con el fin de evitar la dominancia apical y favorecer el llenado del fruto mediante un balance entre el crecimiento reproductivo y vegetativo, tal como lo afirma Ho (1984).

Surya et al. (2006) encontraron mayor altura y área foliar en plantas de tomate a medida que aumentó la disponibilidad de oxígeno en suelos arcillosos. Y se corrobora el hecho de que la respiración de raíces es favorecida por el incremento en la aireación del suelo (Bhattarai et al., 2004).

\section{Masa fresca}

La masa fresca de hojas, tallo y raíz tuvo una correlación inversa y baja con el contenido de arcilla. La masa fresca de tallo y raíz no presentó diferencias estadísticas pero la mejor condición fue $20 \%$ y $10 \%$ de arcilla respectivamente, mientras que la masa fresca de hojas sí presentó diferencias estadísticas $(P<0,05)$, favorecida por $30 \%$ de arcilla (figura 3 ).
La masa seca de hojas y tallo presentaron diferencias significativas, el primero beneficiado por $30 \%$ de arcilla y el segundo por $20 \%$. El peso seco de raíz no presentó diferencias estadísticas, no obstante, un mayor peso dependió del 10\% de arcilla (figura 4). Sin embargo, la correlación con el contenido de arcilla fue de 0,008, -0,3135 y 0,0496 para hojas, tallo y raíz respectivamente, lo cual indica que la arcilla no tiene un efecto claro sobre la masa seca de las plantas de tomate.

La distribución y densidad de raíces determina el volumen de suelo aprovechable para la absorción de agua y nutrientes por la planta (Giulivo y Pitacco, 1997), el cual fue mayor con 10\% de arci1la. Black et al. (1977) y Richards (1983) afirman que a mayor volumen de suelo húmedo existe mayor desarrollo de raíces. Según Honorato et al. (1988) y Ruiz (2000), en las plantas de uva, mientras mayor es el volumen de suelo explorado por el sistema radicular de las plantas, mayor es el crecimiento y desarrollo de la parte aérea y mayor es la productividad de las plantas. Esto no concuerda con la masa de hojas y tallo pero sí con la mayor producción de frutos de tomate.

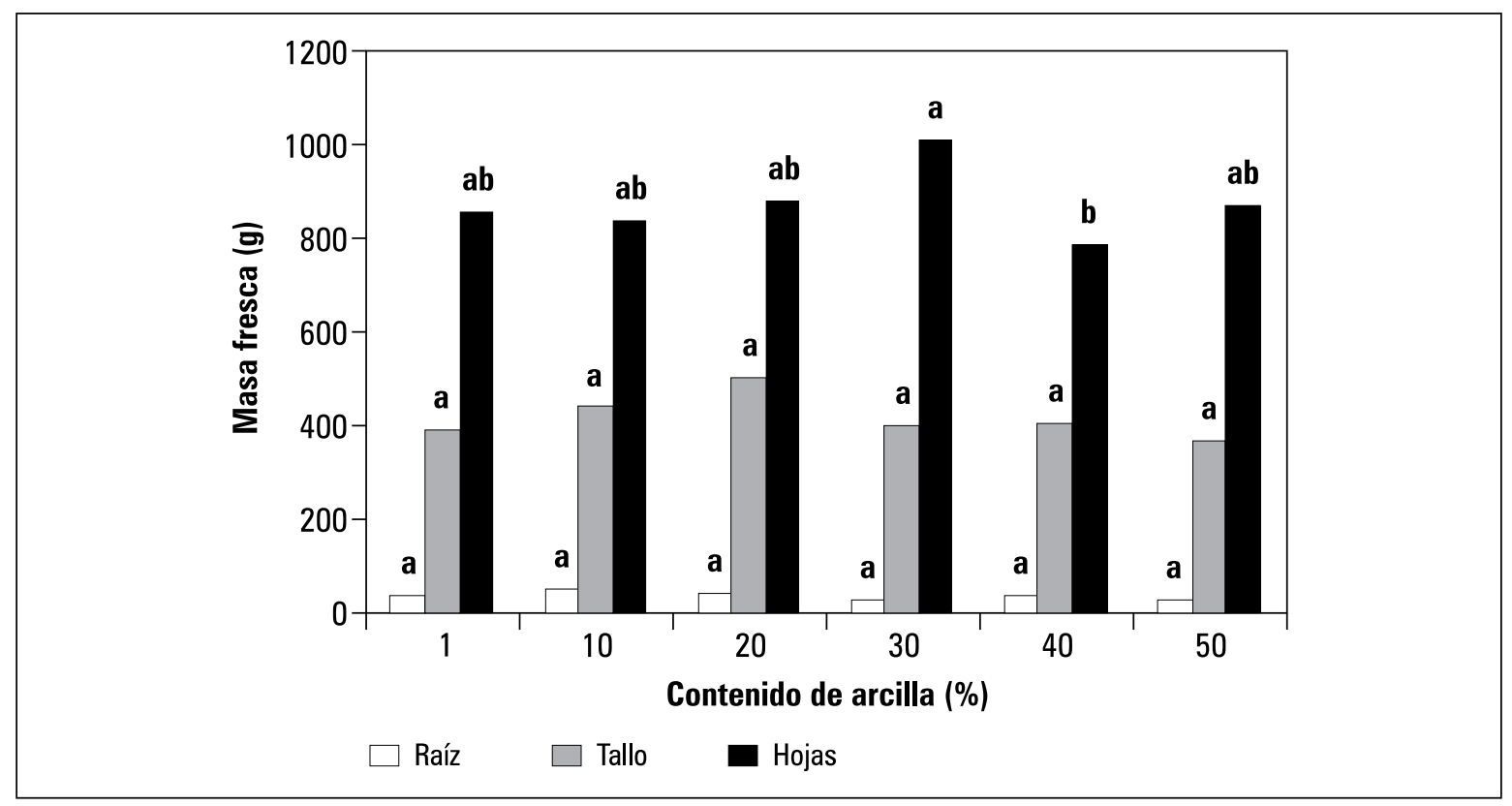

Figura 3. Masa fresca de plantas de tomate larga vida híbrido Granitio bajo el efecto del contenido de arcilla en el suelo. Promedios con letras distintas en la misma serie indican diferencia significativa según la prueba de Tukey $(P \leq 0,05)$. 


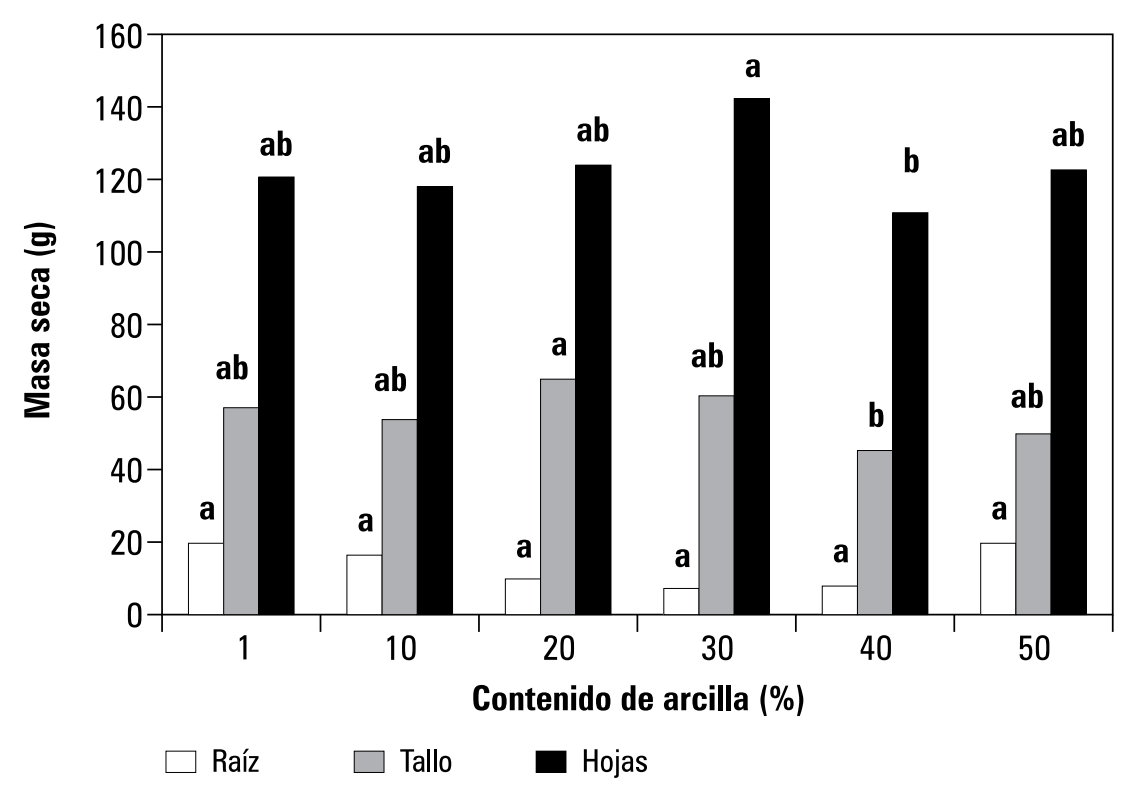

Figura 4. Masa seca de plantas de tomate larga vida híbrido Granitio bajo el efecto del contenido de arcilla en el suelo. Promedios con letras distintas en la misma serie indican diferencia significativa según la prueba de Tukey $(P \leq 0,05)$.

En plantas de tomate de crecimiento indeterminado, la ganancia en masa fresca por parte de los frutos alcanza el $80 \%$ de la masa total (Hurd et al., 1979), debido a que los frutos acumulan más agua que otros órganos similar a lo observado en este estudio, pues cada planta en promedio produjo $3.700 \mathrm{~g}$ de fruto y la masa total promedio fue de $5.000 \mathrm{~g}$, por tanto, el fruto corresponde al $73,4 \%$ de la masa total. No obstante, según Ho (1984) la diferencia en ganancia de masa seca entre órganos es más pequeña. Lo que pone en evidencia que el suministro, almacenamiento y toma de agua por la planta debe ser alto para garantizar un buen llenado de los frutos, no obstante, se pensaba que un alto contenido de arcilla favorecería un buen suministro de agua y fertilizantes, por lo que la función de la fracción arcilla no es tan relevante como sí lo pueden ser la del limo y la arena pues estas favorecen la macroporosidad en el suelo (Malagón y Montenegro, 1990).

En tomate, la producción puede verse disminuida debido a un crecimiento inadecuado del sistema radicular (Ho, 1984), a pesar de que no hubo diferencias en la masa fresca y seca de raíz, sí se observó una menor masa de raíces con el aumento del contenido de arcilla, por esta razón, el suelo con $10 \%$ de arcilla garantiza un buen sistema radicular, pues las raíces de las plantas requieren cantidades adecuadas de oxígeno para su respiración y demás funciones metabólicas en toda la planta (Bhattarai et al., 2006).

\section{CONCLUSIONES}

Las plantas de tomate pueden ser sembradas en suelos con contenidos de arcilla desde $1 \%$ hasta $50 \%$ expresando altos rendimientos. No obstante, el contenido de arcilla del suelo por sí solo no ejerce influencia representativa en el rendimiento de plantas de tomate, aunque afecta la acumulación de masa y el área foliar. A pesar de no presentar diferencias significativas el contenido de arcilla del $10 \%$ presentó mayor correlación con la producción total de tomates y con la calidad extra. Un alto contenido de arcilla en el suelo disminuyó la ganancia en masa fresca y seca de las plantas de tomate. 


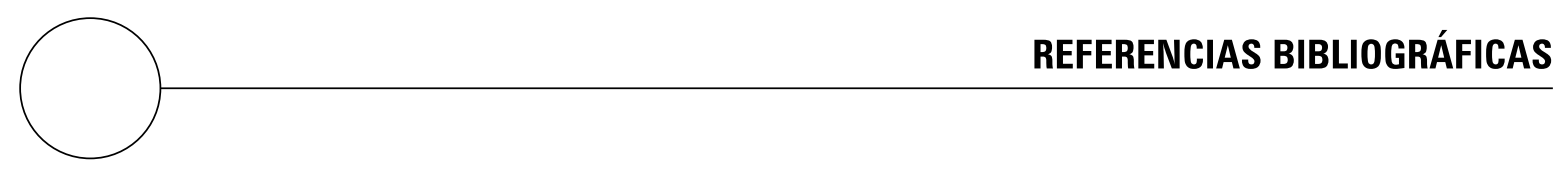

Acharya, C.L.; S.K. Bishnoi y H.S. Yaduvanshi. 1998. Effect of longterm application of fertilizers, and organic and inorganic amendments under continuous cropping on soil physical and chemical properties in an Alfisol. Indian J. Agric. Sci. 58, 509-516.

Agronet. 2009. Área cosechada, producción y rendimiento de tomate. En: Agronet http://www.agronet. gov.co/; consulta: septiembre de 2009.

Ali, M.M. 1998. Degradation of paddy soils during the period 1967-95 in Bangldesh. Ph.D. thesis. Faculty of Life and Environment Sciences, Shimane University, Matsue 690, Japan.

Barrett-Lennard, E.G. 2003. The interaction between water logging and salinity in higher plants: causes, consequences and implications. Plant Soil 253, 35-54.

Bhattarai, S.P.; S. Huber y D.J. Midmore. 2004. Aerated subsurface irrigation water gives growth and yield benefits to zucchini, vegetable soybean and cotton in heavy clay soils. Ann. Appl. Biol. 144, 285-298.

Bhattarai, S.P.; L. Pendergast y D.J. Midmore. 2006. Root aeration improves yield and water use efficiency of tomato in heavy clay and saline soils. Scientia Hort. 108, 278-288.

Black, J.; P.O. Mitchel y P. Newgreen. 1977. Optimum irrigation for young trickle irrigation peach trees. Aust. J. Agric. Anim. Husb. 17, 342-345.

Bohener, J. y F. Bangert. 1988. Effects of fruit set sequence and defoliation on cell number, cell size and hormone levels of tomato fruits (Lycopersicon esculentum Mill.) within a truss. Plant Growth Reg. 7, 141-155.

Bradford, K.J. y S.F. Yang. 1981. Physiological responses of plants to wáter logging. HortScience 16, 25-30.

Brady, N.C. 2004. The nature anual prosperties of soils. $9^{\text {th }}$ ed. Collier, Mac Millan Publishers, New York, NY.

Bresler, E. 1977. Trickle-drip irrigation: principle and application to soil water management. Adv. Agron. 29, 343-393.

Brown, K.W. 1977. Shrinking and swelling of clay, clay strength, and other properties of clay soils and soils. pp. 689-705. En: Dixon, J.B. y S.B. Weed (eds.). Minerals in soil environments. SSSA, Madison, WI.
Bullinger-Weber, G.; L. Reneé-Claire y G. Jean-Michel. 2006. Influence of some physicochemical and biological parameters on soil structure formation in alluvial soils. Eur. J. Soil Biol. 1-14.

Czyzÿ, E. y J. Tomaszewska. 1994. Compaction of a Sandy soil as related to root system and aerial parts development and yields of spring barley. En: Proc. $13^{\text {th }}$ Conf. ISTRO, Aalborg (Denmark) 24(29), 695 698.

Davies, W.J. y J. Zhang. 1991. Root signals and the regulation of growth and development of plants in drying soil. Plant Physiol. 42, 55-76.

Dry, P.R.; B.R. Loveys; M.G. McCarthy y M. Stoll. 2001. Strategic irrigation management in Australian vineyards. J. Int. Sci. Vigne Vin. 35, 129-139.

Escobar, H. y R. Lee. 2001. Producción de tomate bajo invernadero. Cuadernos CIIA, Fundación Universidad de Bogotá Jorge Tadeo Lozano, Chía, Colombia.

Giulivo, C. y A. Pitacco. 1997. Studying the root system of grapevine. Acta Hort. 427,63-66.

FAO. 2007. Faostat. Área cosechada, producción y rendimiento de tomate. En: http://faostat.fao.org/ site/567/DesktopDefault.aspx?PageID = 567\# ancor; consulta: septiembre de 2009.

Fernandes, A.A. 2000. Fontes de nutrientes influenciando o crescimento, a produtividade e a qualidade de tomate, pepino e alface, cultivados em hidroponia. Tesis de maestria, Universidade Federal de Viçosa, Viçosa, Brasil.

Fernandes, A.A.; H.E.P. Martinez y P.C.R. Fontes. 2002. Produtividade, qualidade dos frutos e estado nutricional do tomateiro tipo longa vida conduzido com um cacho, em cultivo hidropônico, em função das fontes de nutrientes. Hort. Bras. 20, 564-570.

Glenn, D.M. 2000. Physiological effects of incomplete root zone wetting on plant growth and their implications for irrigation management. HortScience 35, 1041-1043.

Ho, L.C. 1984. Partitioning of assimilates in fruiting tomateo plants. Plant Growth Reg. 2, 277-285.

Ho, L.C. 1992. Fruit growth an sink strength. pp. 101124. En: Marshal, C. y J. Grace (eds.). Fruit and seed production: aspects of development, environmental 
physiology and ecology. SEB Seminar Series, Cambridge, UK.

Honorato, R.; H. Silva y C. Bonomelli. 1988. Efecto del suelo en el patrón de enraizamiento y productividad de la vid. Cienc. Inv. Agr. 15, 159-169.

Huett, D.O. y E.B. Dettmann. 1988. Effect of nitrogen on growth, fruit quality and nutrient uptake of tomatoes grown in sand. Aust. J. Exp. Agric. 28, 391-399.

Hurd, R.G.; A.P. Gayy A.C. Mountifield. 1979. The effect of partial flower removal on the relation between root, shoot and fruit growth in the indeterminate tomato. Ann. Appl. Biol. 93, 77-89.

INIA-ODEPA. 2000. Proyecto sistemas de validación y transferencia de tecnologías de riego sector regado de las provincias de San Felipe y Los Andes. 52 p. Convenio INIA-ODEPA. Instituto de Investigaciones Agropecuarias, Centro Regional de Investigación La Platina, Santiago, Chile.

Instituto Geográfico Agustin Codazzi (IGAC). 2006. Métodos analíticos de laboratorio de suelos. 6 a ed. Imprenta Nacional, Bogotá.

Kinet, J.M. y M.M. Peet. 1997. Tomato. pp. 259-294. En: Wien, H.C. (ed.). The physiology of vegetable crops. CAB International, Wallingford, CT.

Logendra, L.S. y H.W. Janes. 1999. Hydroponic tomato production: growing media requirements. Acta Hort. 2, 483-486.

Malagón, D. y H. Montenegro. 1990. Propiedades físicas de los suelos. IGAC, Bogotá.

Marschner, H. 2002. Mineral nutrition of higher plants. $2^{\text {nd }}$ ed. Academic Press, London.

Martínez, L. y A. Zinck. 1994. Modelling spatial variations of soil compaction in the Guaviare colonization area, Colombian Amazonian. ITC J. 3, 252-262.

Medina, A.; A. Comman y H. Escobar, 2001. Riego y fertilización. pp. 29-42. En: Escobar, H. y R. Lee (eds.). Producción de tomate bajo invernadero. Cuadernos CIAA, Fundación Universidad de Bogotá Jorge Tadeo Lozano, Chía, Colombia.

Meek, B.D., C.F. Ehlig; L.H. Stolzy y L.E. Graham. 1983. Furrow and trickle irrigation: effects on soil oxygen and ethylene and tomato yield. Soil Sci. Soc. Amer. J. 47, 631-635.
Mejia, C.L. 1975. Characteristics of a common soil toposequence of the Llanos orientales of Colombia. Tesis de maestría. Department of Soil Science, North Carolina State University, Raleigh, NC.

Okano, K.; Y. Sakamoto y S. Watanabe. 2000. Reuse of drainage water for the production of high quality fruits in single-truss tomato grown in a closed hydroponic system. Acta Hort. 511, 277-286.

Peralta, I.E. y D.M. Spooner. 2007. History, origin and early cultivation of tomato (Solanaceae). pp. 1-27. En: Razdan, M.K. y A.K. Mattoo. (eds.). Genetic Improvement of Solanaceous Crops. Vol. 2. Science Publishers, Enfield, UK.

Radin, B.; H. Bergamaschi; C.R. Junior; N.A. Barni; R. Matzenauer e I.A. Didoné. 2003. Eficiência de uso da radiação fotossinteticamente ativa pela cultura do tomateiro em diferentes ambientes. Pesq. Agrop. Bras. 38(9), 1017-1023.

Richards, D. 1983. The grape root system. Hort. Rev. 5, $127-168$

Ruiz, R. 2000. Dinámica nutricional en cinco parrones de diferente productividad del valle central regado de Chile. Agric. Téc. 60, 379-398.

Sakamoto, Y.; S. Watanabe y K. Okano. 2000. Multishoot training in single-truss tomato cultivation. Bull. Natl. Res. Inst. Veg. Ornam. Plants Tea 15, 115-122,

Selles Van Sch, G.; R. Ferreyra y G. Contreras. 2003. Manejo de riego por goteo en uva de mesa $\mathrm{cv}$. thompson seedless cultivada en suelos de textura fina. Agric. Téc. 63(2), 180-192.

Sharma, P.K.; J.K. Ladha; T.S. Verma; R.M. Bhagat y A.T. Padre. 2003. Rice-wheat productivity and nutrient status in a lantana- (Lantana spp.) amended soil. Biol. Fertil. Soils 37(2), 108-114.

Surya, P.; L. Pendergast y D.J. Midmore. 2006. Root aeration improves yield and water use efficiency of tomato in heavy clay and saline soils. Scientia Hort. 108, 278-288.

United States Department of Agriculture (USDA). 2006. Keys to soil taxonomy. $10^{\text {th }}$ ed. Washington, DC. 